\title{
Which psychological interventions work?
}

\author{
A Keen \\ Consultant Health Psychologist, Woolmanhill Hospital and Royal Aberdeen Children's Hospital, Aberdeen, UK
}

\begin{abstract}
It is the daily behaviour of people with diabetes that most influences shorter-term and longer-term health outcomes. Consequently, health professionals involved in diabetes care provision are routinely trying to make sense of, and change, health-related behaviour. The aims of this article were to: a) identify whether or not psychological interventions can lead to improved health outcomes in adults and children, and b) if so, what was the nature of the psychological interventions that worked best. Overall, the literature on young people with types I diabetes and adults with types $I$ and 2 diabetes indicates that psychological interventions are effective in improving glycaemic control in the short term. However, there was insufficient evidence to conclude which psychological interventions were most effective. The literature on psychological interventions in diabetes is relatively underdeveloped and therefore has many limitations.
\end{abstract}

\author{
Correspondence to A Keen, \\ Diabetes Clinic, Woolmanhill \\ Hospital, Skene Street, \\ Aberdeen AB25 ILD, UK \\ a.keen@nhs.net
}

doi:I0.4997/JRCPE.20I0.S03

\section{INTRODUCTION}

Helping adults and children with diabetes to avoid shortand longer-term complications is the primary aim of diabetes services. Unfortunately, just under half of people with diabetes do not attain good control.' There is a general consensus that reducing diabetes-related complications can only occur by improving the ability of people with diabetes to self-care..$^{1-3}$ This is because it is the day-to-day health-related behaviours that are the most important determinant of the outcomes of care, rather than the average of three or so hours per year contact with health professionals that occurs in the UK. ${ }^{3}$ We know that simply telling adults and young people (and their families) what they should do often does not work, and that there is little relationship between knowledge and behaviour. ${ }^{4}$ Therefore, in theory at least, diabetes services are in the business of trying to understand and change health-related behaviours.

There are a range of health-related behaviours which are of interest to diabetes services. These include those behaviours associated with average blood glucose levels, the occurrence of hypoglycaemic and hyperglycaemic episodes, high blood pressure, smoking and obesity. ${ }^{2.5}$ Typically, the behaviours that influence these important clinical indicators are complex and reflect aspects of lifestyle that are long-standing. Furthermore, the relationships among the clinical indicators and behaviours of interest can be bi-directional so establishing, for example, causality can be extremely problematic, especially where glycaemic control and self-care behaviours are concerned.The underlying reasons for peoples' difficulties managing effectively their condition vary. Many of the significant barriers to improved control relate to beliefs that people with diabetes have about themselves and their condition, and relate to emotional well-being. ${ }^{6}$
Psychological theories and models have a long history of informing attempts to change behaviour and improve emotional well-being. Over recent years many clinical guidelines in the UK by the National Institute for Health and Clinical Excellence (NICE) and the Scottish Intercollegiate Guidelines Network (SIGN) have included recommendations for psychological interventions. Evidence-based recommendations have been made not only for the treatment of mental health problems such as depression and anxiety ${ }^{7.8}$ but also for physical health conditions such as low back pain and obesity ${ }^{10}$ and changing behaviour related to public health issues." The aim of this paper is to try to establish whether psychological interventions are effective in improving the short- and longer-term health outcomes of children and adults with diabetes, and if so which specific types of interventions work best.

\section{CHILDREN AND ADOLESCENTS WITH TYPE I DIABETES}

There have been a small number of high-quality systematic reviews of the effectiveness of psychological interventions in young people with type I diabetes. An extensive review on behalf of the National Institute for Health Research's Health Technology Assessment programme (NIHR HTA) was published nearly a decade ago. ${ }^{12}$ This review, which contained a mixture of psychological and educational intervention studies, included 25 randomised controlled trials (RCTs) of which 12 contained sufficient details to allow effect size calculations of changes in glycosylated haemoglobin $\left(\mathrm{HbA}_{\mathrm{c}}\right)$. The mean effect size resulting from the psychological interventions was 0.33 , which translates to a reduction of about $0.6 \%$ in $\mathrm{HbA}_{\mathrm{lc}}$. Eight RCTs contained sufficient data to calculate the mean effect size for psychosocial outcomes, which was 0.37 . 
The authors highlighted a number of weaknesses in the literature. For example, most studies were underpowered to identify the levels of change highlighted above; followup was over a relatively brief period in view of the fact that diabetes is a lifelong condition; most studies were conducted in the USA and most interventions were not theoretically grounded (which generally are less effective than theoretically derived interventions). The reviewers also referred to more clinically relevant issues for example, they could identify no studies which had varied the intervention depending on the actual specific circumstances of participating subjects. In addition, because of small sample sizes, the review was unable to reach any conclusions about the effectiveness of psychological interventions to help those young people with poor control.

Several years later, this systematic review was updated, again including psychological and educational interventions. ${ }^{13}$ Compared with the earlier review, ${ }^{12}$ the authors noted an increased proportion of RCTs (54\% compared with $40 \%$; an increased mean number of subjects per study (79.7 vs 53.8) but no meaningful increase in theoretically based interventions. Nine RCTs published since the 200I review were used to calculate the mean effect size, which was 0.1 I. This substantially lower figure is largely due to three intervention studies (two of which were based on cognitive behavioural therapy principles) resulting in poorer control (negative effect sizes of -0.20 , $-0.1 \mathrm{I}$ and $-0.3 \mathrm{I}$ ). The median effects size on $\mathrm{HbA}_{\mathrm{lc}}$ was similar to the earlier review ( 0.17 vs 0.18$)$, as were the mean and median effect sizes for psychosocial outcomes ( 0.35 vs 0.36 and 0.38 vs 0.37 , respectively). The authors noted that no particular theoretical approach to the design and implementation of psychological interventions appeared to be superior to others. There remained a number of significant limitations in the literature; for example, it was not possible to separate the intervention from the interventionist. That is, it was unclear if similar results would be obtained by others delivering the interventions studied.

A systematic review published in 2005 specifically explored the effectiveness of family-based interventions. ${ }^{14}$ The authors identified 19 RCTs which contained a mixture of psychological and educational interventions. Of these, 12 studies concerned children or adolescents with the pooled effect on $\mathrm{HbA}_{\mathrm{lc}}$ of $0.6 \%$. The fact that both this review and that of the NIHR HTA ${ }^{12}$ several years earlier shared three studies within their analysis cannot account fully for the strikingly similar results. The authors highlighted that the heterogeneity of interventions studied and psychosocial outcome measures used were important weaknesses within the existing literature.

There has been one systematic review of RCTs which included only psychological interventions, rather than those which included both psychological and educational components. $^{15}$ An educational intervention within this review was defined as being specifically designed to increase diabetes-related knowledge and skills, to improve self-management. There is typically an educational component within psychological interventions. However, in this case its purpose is usually to develop psychological sophistication in line with the underlying theory from which the intervention is derived, and then to apply this to defined clinical problems. This review reported a pooled effect size of 0.35 , which is equivalent to a reduction of $0.48 \%$ in $\mathrm{HbA}_{\mathrm{Ic}}$. Of the $10 \mathrm{RCT}$ s included in this calculation, six were included in the $200 \mathrm{I}$ review. ${ }^{12}$ The authors further noted an improvement in psychosocial outcomes of mean effect size to 0.46 . Moreover, they highlighted the fact that the quality of studies was poor to moderate, and that most interventions were based broadly on cognitive behavioural principles. Comparison therefore between different types of psychological interventions was not possible.

\section{ADULTS WITH TYPE I DIABETES}

There are fewer systematic reviews of psychological interventions used with adults than with young people. However, we do have one high-quality, relatively recent systematic review and meta-analysis which helpfully excluded educational interventions. ${ }^{15}$ This review identified II RCTs and these were used to calculate the standardised mean effect size of 0.17. Of these II studies, eight resulted in improved glycaemic control and three in poorer control. The mean effect size for improvement in psychological distress was 0.35 . Although most of the studies reviewed were informed by cognitive behavioural principles they were certainly not homogeneous. For example, one RCT evaluated the effect of two I5-minute individual exploratory discussions about well-being with diabetes specialist nurses against standard care in the Netherlands. ${ }^{16}$ Another RCT compared the effectiveness of cognitive behavioural techniques against blood glucose awareness training, with both interventions delivered in six two-hour sessions to groups of subjects. ${ }^{17}$ Yet another compared the effect of an intensive in-patient progressive muscle relaxation programme against treatment, as usual in the USA. ${ }^{18}$

An RCT published subsequently to the above review is worthy of note. A relatively large RCT in the UK compared the effects of about four hours of motivational enhancement therapy over two months versus four hours of motivational enhancement therapy plus eight hours of cognitive behavioural therapy (CBT) over six months versus usual care. ${ }^{19}$ Unusually, each arm of this study had more than 100 subjects. Twelve months later, the motivational enhancement therapy plus $\mathrm{CBT}$ group had a mean $\mathrm{HbA}_{\mathrm{lc}}$ of nearly $0.5 \%$ lower than subjects who had received usual care. Arguably the only potential difficulty with this study is the training of the nurses who delivered the interventions. The authors detail that they 
A Keen

sensibly continually assessed nurses' skills during the training period, allowing them to deliver the study intervention only when deemed sufficiently competent. However, the training period was only three months, and this is significantly shorter (by at least six months) than even those training courses designed for delivery of lowintensity interventions based on CBT (generally guided self-help), and we certainly know that outcomes are linked to therapists' skill levels in CBT.?

\section{ADULTS WITH TYPE 2 DIABETES}

As far as the author is aware, there has been one systematic review of psychological interventions to improve glycaemic control among adults with type 2 diabetes. A review published in $2004^{20}$ identified 12 RCTs, of which nine resulted in better control and three in poorer control. The standardised mean effect size was 0.32 in favour of psychological interventions, which the authors indicate equates to a decrease of $0.76 \%$ in $\mathrm{HbA}_{\mathrm{Ic}}$. Removing two studies wherein the control groups received less intensive psychological interventions resulted in an increased effect size of 0.44 and a corresponding drop in $\mathrm{HbA}_{\mathrm{lc}}$ of $1.06 \%$. Five RCTs could be used to calculate the mean effect size for improvement in psychological distress, which was 0.58. It is important to note that psychological difficulties (such as depression, anxiety and binge eating) were explicitly a target for the interventions.

It is clear from this review that the target groups have been relatively dissimilar. For example, some studies specifically targeted people with type 2 diabetes who also had clinical depression, a binge eating problem or suboptimal control or were obese.These are very different groups and certainly within a cognitive behavioural paradigm would require dissimilar management and treatments. The authors highlighted the fact that most interventions (10 of the 12) used were based on based on cognitive behavioural theory and interventions. It is extremely difficult to find terms to encompass the nature of the psychological interventions that have been used in studies in diabetes, because of their diversity. It is unlikely, however, that many within the cognitive behavioural therapy community would consider 16 Qigong relaxation training sessions by a Qigong doctor as an example of their work. This is not meant to be a criticism of the review, rather a comment to emphasise the fact that we need to be mindful of the homogeneity of the interventions and interventionists.

Subsequent to the 2004 review, ${ }^{20}$ there had been one RCT worth highlighting. A US study ${ }^{21}$ compared a standard seven-hour education programme provided to newly diagnosed low socioeconomic status adults with type 2 diabetes $(n=38)$, and a four-hour condensed version of the educational programme plus three hours of a psychological intervention $(n=43)$. The intervention was based on an approach called acceptance and commitment therapy (ACT). Acceptance and commitment therapy is part of the so-called third wave of psychological treatments. It emphasises the development of mindfulness (trying to live in, and focus on, the present moment), acceptance (including the fact that difficulties are inevitable) and valuebased living (so you try to live an interesting and meaningful life, even in the face of difficulties).

There were a number of significant results between the groups across the pre-treatment to follow-up point three months later. The mean $\mathrm{HbA}_{\mathrm{lc}}$ in the control group fell from $8.21 \%$ to $8.07 \%$, while in the ACT group it decreased from $8.17 \%$ to $7.47 \%$, significantly favouring the latter group. Similarly, in the control group the number of subjects whose $\mathrm{HbA}_{\mathrm{Ic}}$ was below $7 \%$ decreased slightly from 10/38 to $9 / 38$ between pre-treatment and follow-up. In the ACT group over the same period there was an increase from I I/43 to 21/43. Again the difference between the two groups was significant. The main weaknesses of this study are that the number of subjects is relatively small, the follow-up period short and the ACT intervention was delivered by a single interventionist.

\section{DISCUSSION}

It is clear that the existing evidence suggests that psychological interventions are effective in improving the short-term glycaemic control of children and adults with type I diabetes and adults with type 2 diabetes. The extent to which this is the case is less clear, and we have no evidence of whether gains are maintained over longer periods. Expecting an improvement in the region of about $0.5 \%$ in $\mathrm{HbA}_{\mathrm{lc}}$ would appear to be realistic. There is no substantial evidence to help inform us which of the many possible psychological interventions available are most effective overall and also where specific sub-groups are concerned. For example, we currently have little to guide us on what is the most effective psychological intervention for younger children, adolescents, those with type I diabetes, those with type 2 diabetes, those with especially poor control, and so on.

The existing literature is beset with limitations. However, it is worth reflecting on the fact that a recent update of a UK national clinical guideline on the management of depression using psychological interventions resulted in the identification of 139 RCTs, which in total included data on nearly 13,000 subjects. ${ }^{7}$ This current review highlights the difficulty of systematically evaluating literature on a topic in its infancy. In short, in the area of diabetes we have relatively little data typically provided from smallscale studies wherein the psychological interventions are relatively idiosyncratic, the extent and nature of the educational components are often unclear and inclusion criteria is broad. The latter point profoundly influences effect size calculations and may in part account for the considerably lower effect size values for psychosocial outcomes when compared to the mental health literature (which is typically in the region of 0.75 ). 
Unfortunately, understanding exactly what is being described by the term 'psychological intervention' is not easy, particularly for those with no training in professional psychology. There are myriad psychological theories and models which can be used to inform the design and delivery of interventions. Some are clearly dissimilar. For example, psychoanalysis is typically a long-term exploratory approach which seeks to resolve unconscious conflicts, and CBT is a time-limited, problem-orientated approach that seeks to help change unhelpful ways of thinking and behaving. However, even CBT is a broad church which encompasses many somewhat different approaches. ${ }^{22}$ This means that almost all studies are not evaluating a specific approach (such as CBT), rather more usually a small number of affiliated techniques. This in turn results in the heterogeneity highlighted in the section on type I diabetes in adults.

In view of the problems ascertaining exactly what is being delivered in studies using psychological interventions (and their apparent idiosyncratic nature) it is unsurprising that there is heterogeneity in the psychosocial outcome measures employed. Few studies used the same psychosocial outcome measure and this makes direct comparison problematic, which is especially unhelpful when these variables are hypothesised to act as mediating factors of blood glucose control. For example, it might be the case that researchers hypothesise that aspects of family functioning influence the glycaemic control of children with type I diabetes. However, if all studies investigating this used dissimilar psychosocial measures then comparisons of results are difficult. Indeed, one review $^{13}$ noted that 40 different psychosocial measures were used in the studies they included, with only five being used in more than one RCT. Likewise, the dissimilar terms used to describe interventions which may share many features, such as behavioural family systems therapy, ${ }^{23}$ family therapy ${ }^{24}$ and multisystemic therapy, ${ }^{25}$ do not easily allow replication or clarification of what was actually delivered. Although there have been attempts to dissect psychological interventions into discrete components, ${ }^{26}$ this level of analysis is largely absent within the wider literature on psychological interventions and definitely within the diabetes literature.

An especially difficult issue in the current literature is that we do not know the relative contribution of the intervention itself (the content) and the interventionist to results. This is a commonly occurring theme in the general literature on psychological therapy and behavioural change. Certainly it is the case that there is good evidence that some psychological therapists obtain significantly better or poorer outcomes than others, and these differences can be larger than the effects of using different types of intervention. ${ }^{27}$ This is called the therapist effect.
Another, related, issue highlighted by one systematic review $^{13}$ is that RCTs had typically compared interventions to routine care and therefore it was not possible to separate the influence of increased contact with a diabetes professional per se.This again is a long-standing topic of discussion and exploration, and cannot be easily disentangled from the so-called therapist effects highlighted above because personal characteristics that facilitate relationship-building are associated with better outcomes. ${ }^{28}$ Overall, the empirical literature indicates that psychological interventions are superior to placebo control conditions, which are in turn superior to doing nothing. ${ }^{27}$ To explore both therapist effects and the influence of actual increased contact time would require considerably more sophisticated (and expensive) research designs than have been conducted up to this point.

Finally, it is worth noting that the RCTs conducted over the past couple of decades do not reflect standard clinical practice in the UK. For example, the subjects in the literature on young people are not representative of those who would usually receive formal psychological interventions and the interventions themselves are also dissimilar. Generally, studies have used broad inclusion criteria so the young people were often neither clinically distressed nor poorly controlled. However, dedicated psychology provision to diabetes services is limited, if present at all. 29,30 Therefore, as psychologists are a precious resource, diabetes teams tend to refer the neediest young people, usually those with clinically significant psychological problems and the poorest control. Consequently, little psychology time is dedicated specifically to improving the glycaemic control of the general diabetes population. ${ }^{29}$

Those young people with diabetes referred to psychologists in the UK would ordinarily receive an individual assessment which would inform the exact nature of the psychological intervention. That is, all young people with diabetes referred to local NHS psychology services would not routinely receive the same intervention, even if seen by the same psychologist. The situation is similar in psychology service provision to adults with diabetes, with the additional difficulty that there is considerably less of it and the number of patients is vastly increased. ${ }^{29}$ 
A Keen

\section{REFERENCES}

I Scottish Executive. Scottish diabetes framework. Edinburgh: Scottish Executive, 2006

2 National Institute of Health and Clinical Excellence. Type 2 diabetes: national clinical guideline for management in primary and secondary care (update). London: NICE; 2008. Available from: http:// guidance.nice.org.uk/CG66/Guidance/pdf/English

3 Department of Health. Self care - a real choice: self care - a practical option. London: Department of Health; 2005.

4 Knight KM, Dornan T, Bundy C.The diabetes educator: trying hard, but must concentrate more on behaviour. Diabet Med 2006; 23:485-501. doi:I0.1III/j.1464-549I.2005.01802.x

5 National Institute of Health and Clinical Excellence. Type I diabetes in adults: national clinical guideline for diagnosis and management in primary and secondary care (update). London: NICE; 2004. Available from: http://www.nice.org.uk/nicemedia/pdf/ CG015_fullguideline_adults_development_section.pdf

6 Skinner TC. Psychological barriers. Eur J Endocrinol 2004; I5 I:T I3TI7. doi:I0.1530/eje.0.15IT0I3

7 National Institute of Health and Clinical Excellence. Depression in adults (update): depression: the treatment and management of depression in adults. London: NICE; 2009. Available from: http:// www.nice.org.uk/nicemedia/pdf/Depression_Update_FULL_ GUIDELINE.pdf

8 National Institute of Health and Clinical Excellence. Clinical guidelines for the management of anxiety: management of anxiety (panic disorder, with or without agoraphobia, and generalised anxiety disorder) in adults in primary, secondary and community care. London: NICE; 2004. Available from: http://www.nice.org.uk/nicemedia/pdf/ cg022fullguideline.pdf

9 National Institute of Health and Clinical Excellence. Low back pain early management of persistent non-specific low back pain. London: NICE; 2009. Available from: http://www.nice.org.uk/nicemedia/pdf/ CG88fullguideline.pdf

10 National Institute of Health and Clinical Excellence. Obesity: the prevention, identification, assessment and management of overweight and obesity in adults and children. London: NICE; 2007. Available from: http://www.nice.org.uk/nicemedia/pdf/CG43FullGuidelinel.pdf

II National Institute of Health and Clinical Excellence. Behaviour change at population, community and individual levels. London: NICE: 2007. Available from: http://www.nice.org.uk/nicemedia/pdf/ PH006guidance.pdf

12 Hampson SE, Skinner TC, Hart J et al. Effects of educational and psychosocial interventions for adolescents with diabetes mellitus: a systematic review. Health Technol Assess 200 I; 5:1-79.

13 Murphy HR, Rayman G,SkinnerTC.Psycho-educational interventions for children and young people with type I diabetes. Diabet Med 2006; 23:935-43. doi:10.1IIII/j.1464-549I.2006.01816.x

14 Armour TA, Norris SL, Jack $L$ et al. The effectiveness of family interventions in people with diabetes mellitus: a systematic review. Diabet Med 2005; 22:1295-305. doi:I0.IIII/j.1464-549I.2005.01618.x

15 Winkley K, Ismail K, Landau $S$ et al. Psychological interventions to improve glycaemic control in patients with type I diabetes: systematic review and meta-analysis of randomised controlled trials. BMJ 2006; 333: 65-9. doi:10.1 I36/bmj.38874.652569.55

16 Pouwer F, Snoek, FJ, Van der Ploeg HM et al. Monitoring of psychological well-being in outpatients with diabetes: effect on mood, HbAlc, and the patient's evaluation of the quality of diabetes care: a randomized controlled trial. Diabetes Care 200I; 24:1929-35. doi:10.2337/diacare.24.II.1929
17 Van der Ven NC, Hogenelst MH, Tromp-Wever AM et al. Shortterm effects of cognitive behavioural group training (CBGT) in adult Type I diabetes patients in prolonged poor glycaemic control. A randomized controlled trial. Diabet Med 2005; 22:1619-23. doi:I0.1III/j.1464-549I.2005.0169I.x

18 Feinglos MN, Hastedt P, Surwit RS. The effects of relaxation therapy on patients with type I diabetes mellitus. Diabetes Care 1987; 10:72-5. doi:10.2337/diacare. 10.1.72

19 Ismail K, Thomas SM, Maissi E et al. Motivational enhancement therapy with and without cognitive behavior therapy to treat type I diabetes: a randomized trial. Ann Intern Med 2008; 149:708-19.

20 Ismail K, Winkley K, Rabe-Hesketh S. Systematic review and metaanalysis of randomised controlled trials of psychological interventions to improve glycaemic control in patients with type 2 diabetes. Lancet 2004; 363:1589-97. doi:10.1016/S0I406736(04) 16202-8

21 Gregg JA, Callaghan GM, Hayes SC et al. Improving diabetes self management through acceptance, mindfulness, and values: a randomized controlled trial. J Consult Clin Psychol 2007; 75:336-43. doi:10.1037/0022-006X.75.2.336

22 British Association for Behavioural \& Cognitive Psychotherapies. What is CBT? Bury: BABCP; 2007. Available from: http://www. babcp.com/public/what-is-cognitive-behaviour-therapy/

23 Wysocki T, Harris MA, Buckloh LM et al. Randomized trial of behavioral family systems therapy for diabetes: maintenance of effects on diabetes outcomes in adolescents. Diabetes Care 2007; 30:555-60. doi:10.2337/dc06-1613

24 Rydén O, Nevander L, Johnsson P et al. Family therapy in poorly controlled juvenile IDDM: effects on diabetic control, selfevaluation and behavioural symptoms. Acta Paediatr 1994; 83:28591. doi:10.IIII/j.165I-2227.1994.tb/8096.x

25 Ellis DA, Yopp J, Templin T et al. Family mediators and moderators of treatment outcomes among youths with poorly controlled type I diabetes: results from a randomized controlled trial. J Pediatr Psychol 2007; 32: 194-205. doi:I0.1093/jpepsy/jsjl I6

26 Abraham C, Michie S.A taxonomy of behavior change techniques used in interventions. Health Psychol 2008; 27:379-87. doi:10.1037/0278-6133.27.3.379

27 Lambert M, Ogles B.The efficacy and effectiveness of psychotherapy. In:Lambert MJ, editor. Bergin and Garfield's handbook of psychotherapy and behavior change. 5th ed. New York: John Wiley \& Sons; 2004. p. I30-93.

28 Beutler LE, Malik, M, Alimobamed S et al. Therapist variables. In: Lambert MJ, editor. Bergin and Garfield's handbook of psychotherapy and behavior change. 5th ed. New York: John Wiley \& Sons; 2004. p. 227-306.

29 Psychology Working Group. A review of psychology provision to adults \& children with diabetes in Scotland. Edinburgh: Diabetes in Scotland; 2006. Available from: http://www.diabetesinscotland.org uk/Publications/SDG\%20Psychology\%20report\%202006.pdf

30 Edge JA, Swift PGF, Anderson W et al. Diabetes services in the UK: fourth national survey; are we meeting NSF standards and NICE guidelines? Arch Dis Child 2005; 90:1005-9. doi:10.1136/ adc.2005.071613 